\title{
Energy gap in superconducting fullerides: optical and tunneling studies.
}

\author{
Daniel Koller, Michael C. Martin and László Mihály \\ Department of Physics, SUNY at Stony Brook, Stony Brook, NY 11794-3800 \\ György Mihály甘, Gábor Oszlány迷, Gabriel Baumgartner and László Forró \\ Department of Physics, Ecole Polytechnique Federale de Lausanne CH-1015 Lausanne, Switzerland
}

(September 20, 2021)

Tunneling and optical transmission studies have been performed on superconducting samples of $\mathrm{Rb}_{3} \mathrm{C}_{60}$. At temperatures much below the superconducting transition temperature $T_{\mathrm{c}}$ the energy gap is $2 \Delta=5.2 \pm 0.2 \mathrm{meV}$, corresponding to $2 \Delta / k_{B} T_{\mathrm{c}}=4.2$. The low temperature density of states, and the temperature dependence of the optical conductivity resembles the BCS behavior, although there is an enhanced "normal state" contribution. The results indicate that this fulleride material is an s-wave superconductor, but the superconductivity cannot be described in the weak coupling limit.

PACS: $74.70 . \mathrm{Wz} 74.25 . \mathrm{Gz} 74.50 .+\mathrm{r}$

The symmetry of the order parameter and the magnitude of the energy gap are two of the most fundamental properties of superconductors. While there is a general agreement about the $s$-wave nature of superconductivity in the alkali metal fulleride compounds, the magnitude of the energy gap is not known with sufficient accuracy.

Why is the determination of the energy gap so important? Let us consider, for example, the Eliashberg theory of phonon-mediated superconductivity, and represent the phonons by a single Einstein mode. [1, 2 There are two parameters in this theory: The phonon frequency $\omega_{0}$ and the coupling between electrons and phonons $\lambda$. (The latter one may be viewed as an "effective" electron - phonon coupling constant, incorporating the "true" $\lambda$ and the electron - electron repulsion parameter $\mu$. [1, 3, 3 ) In the weak coupling (BCS) limit the quantity $\omega_{0} \exp (-1 / \lambda)$ is the only relevant combination of parameters. In that limit the ratio of the low temperature value of the superconducting energy gap to the critical temperature is always $2 \Delta / k_{B} T_{\mathrm{c}}=3.53$. If the coupling is strong, however, knowledge of the critical temperature and the low temperature gap provides us with $\lambda$ and $\omega_{0}$. Once the magnitudes of these parameters are known, theoretical models for the microscopic mechanism of superconductivity [4] can be placed in the proper context.

A review of the literature reveals a great disparity between the various measurements of the superconducting energy gap in $\mathrm{Rb}_{3} \mathrm{C}_{60}$. Direct spectroscopic methods to determine the gap include optical spectroscopy, tunneling and photoemission. The first optical study by Rotter et al. [5] resulted in values of $\eta \equiv 2 \Delta / k_{B} T_{\mathrm{c}} \approx 3-4$. Fitzgerald et al. [6] obtained $\eta \approx 3-5$ from transmission measurements on thin film samples. In more recent reflectance measurements on polycrystalline [7] and single crystal 8 samples, Degiorgi et al. obtained $\eta \approx 2.98$ and $\eta \approx 3.45$, respectively. Zhang et al. [9] reported tunneling measurements yielding a value greater than 5 , whereas Jess and co-workers measured values between 2 and 4 in an STM study [10]. Photoemission experiments by the Argonne-University of Illinois collaboration 11 led to $\eta \approx 4.1 \pm 0.4$. Nuclear magnetic resonance $[12.13$ and muon spin relaxation [14] yielded values compatible with the BCS weak coupling limit. (All numerical values summarized here apply to $\mathrm{Rb}_{3} \mathrm{C}_{60}$.)

An accurate determination of $\eta$, and the detailed fit of the theoretical curves to the experimental results answers a fundamental question: Is the coupling between the electrons weak enough for the BCS theory to describe superconductivity? In this Letter we report optical transmission and tunneling measurements unambiguously demonstrating that the ratio $2 \Delta / k_{B} T_{\mathrm{c}}$ is $4.2 \pm 0.2$, significantly larger than the BCS value. Since the weak coupling conditions are not satisfied, we are able to deduce the phonon coupling constant and the characteristic phonon frequency, obtaining $\lambda=0.9$ and $\omega_{0}=210 \mathrm{~cm}^{-1}$ $\left(\hbar \omega_{0}=26 \mathrm{meV}\right)$. The electron - phonon coupling is consistent with the value determined from DC resistivity measurements [15]; the phonon frequency is close to the lowest lying Raman frequency of the $\mathrm{C}_{60}$ molecule [16].

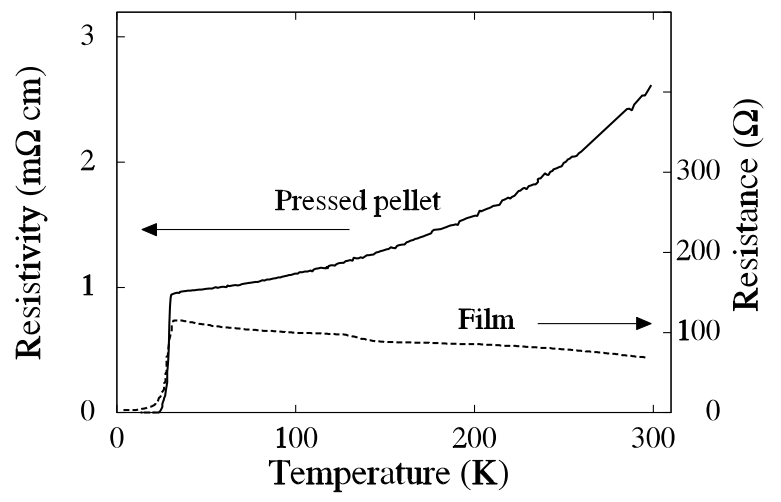

FIG. 1. Resistivity and resistance of the thin film and pressed pellet samples used in the optical and tunneling measurements, respectively.

The pressed pellet samples for the tunneling measurements were made by heating and mixing stoichiometric amounts of $\mathrm{Rb}$ and $\mathrm{C}_{60}$ in a quartz tube for several weeks. According to powder X-ray diffraction, the sample con- 
tained no secondary phases. The material was pressed into a pellet, with four gold wires embedded for electrical contacts. The resistivity as a function of the temperature is shown in Figure 1. The resistivity of the pressed pellet sample was as low as the resistivity of single crystals [15]. The superconducting transition temperature was $30 \mathrm{~K}$.

The optical measurements were performed on samples prepared by deposition of a thin $\mathrm{C}_{60}$ film on a $\mathrm{Si}$ substrate and subsequent exposure to $\mathrm{Rb}$ vapor in a sample cell mounted in a Bomem MB-155 spectrometer. [17] The composition of the sample was monitored by observing the $F_{1 u}(4)$ vibrational mode of the $\mathrm{C}_{60}$ molecule [18]. The thickness of the film was $1.0 \pm 0.1 \mu \mathrm{m}$. Scanning force microscope images of the sample surface showed an irregular surface with poorly matching crystal faces. From room temperature to $30 \mathrm{~K}$ there was a factor of two increase of resistance, typical of thin film samples [19] and indicative of granular morphology (Figure 11). The superconducting transition occurred at 30K; a smearing of the transition suggested a distribution of critical temperatures, also consistent with granular morphology.

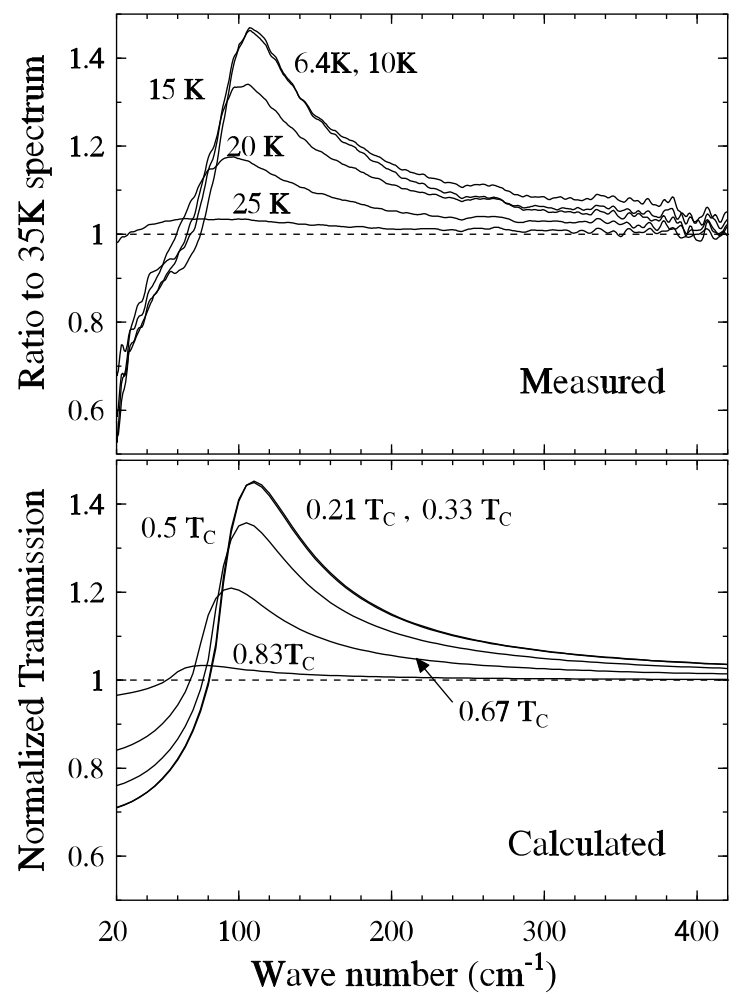

FIG. 2. (top) Far infrared transmission of the sample at temperatures below the superconducting transition. The curves are normalized to the transmission at $T=35 \mathrm{~K}$ in order to eliminate the features due to the silicon substrate. (bottom) Calculated transmission curves, based on dirty limit BCS results.

The far-IR transmission spectra were measured as a function of temperature at the U4IR beamline of the National Synchrotron Light Source, at Brookhaven Na- tional Laboratory. The sample cell was mounted on a Helitran He flow refrigerator and the spectra were taken in a Nicolet 20F Rapid Scan FTIR spectrometer. Due to the capacitive inter-grain coupling, AC measurements (including the optical transmission) are expected to be less sensitive to the grain boundaries then the DC transport. In accordance with this expectation, the optical transmission of the film decreased upon cooling, corresponding to the increasing intrinsic conductivity of the crystallites.

The upper panel in Figure 2 shows the low temperature transmission results, normalized to the spectrum taken in the normal state at $35 \mathrm{~K}$. The pronounced peak in the optical transmission is a direct evidence for the sharp energy gap in the real part of the optical conductivity at $\hbar \omega=2 \Delta$. This feature was first observed for superconductors, and discussed in detail, by Glover and Tinkham [20].

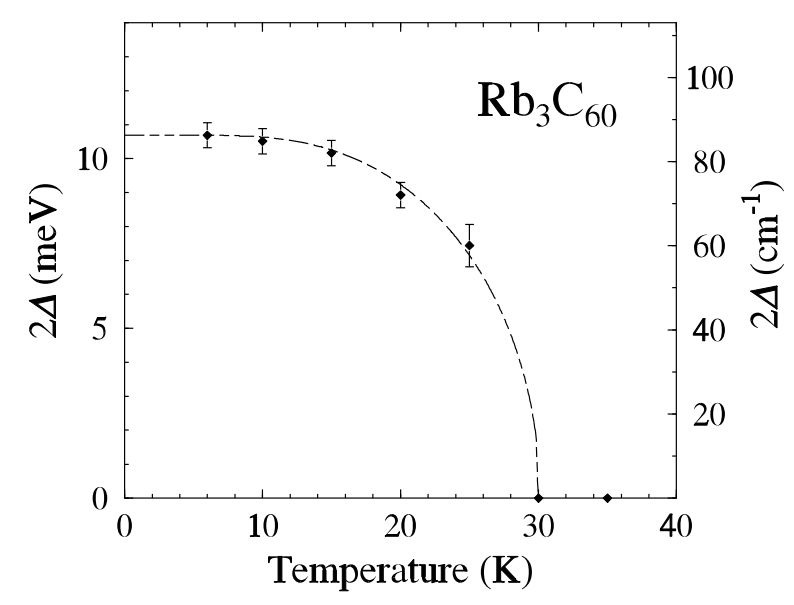

FIG. 3. Temperature dependence of the energy gap evaluated from the optical transmission data. The dashed line represents the BCS temperature dependence, scaled to satisfy $2 \Delta=4.1 k_{B} T_{\mathrm{c}}$.

Calculated transmission curves are shown in the lower panel of Figure 2. In the absence of readily available Eliashberg results for the density of states and optical conductivity, we modified the BCS result 21] to include the effects of inelastic pair breaking [25] in a phenomenological way. The total optical transmission $t$ was represented in a two component model, in the form of $t=w t_{s c}+(1-w) t_{n}$, where $t_{n}$ is the transmissions of a Drude metal, and $t_{s c}$, was calculated from the MattisBardeen [21] conductivity of a superconductor. This approach also accounts for the inevitable imperfections in the sample. In the best fits the weight factor was about $w=30 \%$ at the lowest temperature, and it decreased as the temperature approached $T_{\mathrm{c}}$. The energy gap as a function of the temperature, evaluated from these fits, is shown in Figure 3. Notice that the energy gap at zero temperature corresponds to $\eta \approx 4.1 \pm 0.2$. For comparison, the temperature dependence of the weak coupling 
(BCS) gap, scaled to $2 \Delta=4.1 k_{B} T_{\mathrm{c}}$, is also shown in the Figure.

For the tunneling measurements the pressed pellets were first cooled to low temperature. A break junction was made and controlled by mechanical means. The device was similar to the one used in the tunneling studies of high $T_{\mathrm{c}}$ superconductors [22]. The sample was in $\mathrm{He}$ atmosphere to prevent the degradation of the material due to the chemical reaction with oxygen. The differential conductance of a typical junction at $T=4.2 \mathrm{~K}$ is shown in Figure 1 . The poor thermal stability of the junction prevented us from measuring the temperature dependence of the tunneling.

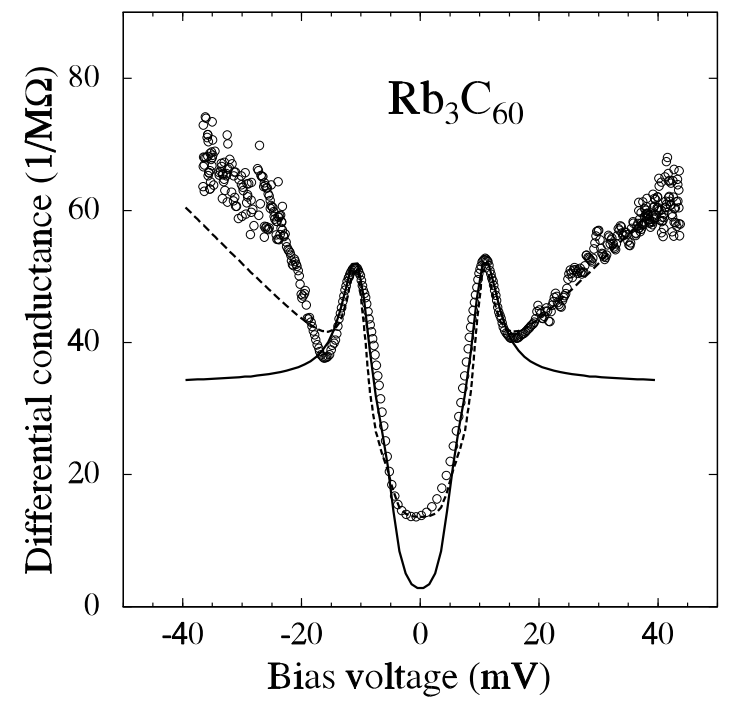

FIG. 4. Differential conductance of the break junction made of a polycrystalline $\mathrm{RbC}_{60}$ pellet (open circles). The continuous line is a three parameter fit using a modified BCS formula for the density of states (see text). The dashed line was obtained by including an additional leakage conductance at zero bias, and the voltage dependence of the transmission function of the junction.

Ideal break junctions are superconductor - insulator superconductor (SIS) junctions. The tunneling current is described by $I \propto \int g f g^{\prime} f^{\prime} \mathrm{d} E$ where $g=g(E)$ and $g^{\prime}=g(E-e V)$ are the densities of states and $f=f(E)$ and $f^{\prime}=f(E-e V)$ are Fermi functions [23]. The simple BCS density of states is often modified to

$$
g(E) \approx\left|\operatorname{Re} \frac{E+\mathrm{i} \delta}{\sqrt{(E+\mathrm{i} \delta)^{2}+\Delta^{2}}}\right|
$$

where the phenomenological parameter $\delta$ accounts for the pair breaking effects [25,26. The continuous line in Figure 1 was obtained by adjusting $\Delta, \delta$ and the vertical scale factor of the differential conductance. Apart from the elevated zero bias conductance and the upturn of conductivity at larger bias voltages, the agreement is satisfactory. (The parameters are $2 \Delta \approx 5.2 \mathrm{meV}$ and $\delta \approx 1.5 \mathrm{meV}$ ). A more complete calculation, including an additional leakage conductance at zero bias, and a voltage dependent tunneling rate [27], results in an even better fit (dashed line). Most importantly, the Figure demonstrates that the value of the energy gap is not sensitive to the processing of the data: the peak position of the raw data, and the two fitting procedures yield $2 \Delta \approx 5.2 \mathrm{meV}$, corresponding to $\eta \approx 4.3 \pm 0.2$

The accuracy of the results and the agreement between the two principal spectroscopic methods (optics and tunneling) leave little doubt about the magnitude of the low temperate energy gap in $\mathrm{Rb}_{3} \mathrm{C}_{60}$. The ratio $\eta \equiv 2 \Delta / k_{B} T_{\mathrm{c}} \approx 4.2 \pm 0.2$ is obtained, which is clearly beyond the weak coupling value. This result is compatible with, although more accurate than, most of the earlier spectroscopic studies [24], and it agrees particularly well with the photoemission study [11]. Thus all three direct methods (tunneling, optics and photoemission) yield very similar values.

In early NMR measurements the absence of the HebelSlichter (H-S) peak in the relaxation rate was interpreted as an evidence for strong coupling. 12,28] More recently, Stenger et al. 13] observed the H-S peak, and argued that $\mathrm{Rb}_{3} \mathrm{C}_{60}$ is in the weak coupling regime. In particular, $\eta \approx 4.8$ was excluded as too high. However, due to the many factors influencing the magnitude of the H-S peak, smaller values of $\eta$ could not be excluded. Interestingly, Stenger et al. puts a lower bound of $200 \mathrm{~cm}^{-1}$ on the phonon frequency, and our result is entirely compatible with that constraint.

Kiefl et al. determined $\eta \approx 3.6 \pm 0.3$ from muon spin rotation experiments. The interpretation of the results is similar to the NMR relaxation rate, except the low temperature limiting behavior is more accessible with muons. The spin relaxation rate depends on the number of electrons in the conduction band. For BCS superconductors these electrons are thermally activated, thus the relaxation rates should exhibit Arrhenius temperature dependence at low $T$. This straightforward interpretation becomes rather complicated for intermediate and strong coupling. At finite temperatures, the density of states is non-zero at the Fermi level (unlike the BCS limit). 25] Indeed, Kiefl et al. used a broadened density of states, such as described in Eq. 11. The value of the gap depends on the assumptions made for the temperature dependence of $\delta$. We believe that in this case it is crucial to use the full Eliashberg calculation to obtain a more reliable value for $\Delta$. Notice the contrast to the direct spectroscopic studies, where the phenomenological parameter $\delta$ does not influence the numerical magnitude of the gap in any significant way.

The dimensionless electron - phonon coupling constant and the characteristic phonon frequency can be determined as follows. According to Carbotte [2], for various superconductors $\eta$ can be well approximated by

$$
\eta \approx 3.53\left(1+12.5\left(\frac{k_{B} T_{\mathrm{c}}}{\hbar \omega_{0}}\right)^{2} \ln \frac{\hbar \omega_{0}}{2 k_{B} T_{\mathrm{c}}}\right) .
$$


The $\eta$ value determined here corresponds to $T_{\mathrm{c}} / \omega_{0}=$ 0.10 . This, in turn, leads to the dimensionless coupling constant of $\lambda=0.89$ (from Eq. 2 of Allen and Dynes [1]) or a slightly larger value if the repulsive electron electron interaction is also considered (one obtains, for example, $\lambda=1.16$ for $\mu=0.1$ ).

Two important conclusions can be drawn from our results. First, the coupling constant is reasonably close to the $\lambda_{t r} \approx 0.65-0.80$, obtained from the DC electrical transport measurements by Vareka and Zettl [15]. Notice that for most BCS superconductors $\lambda$ and $\lambda_{t r}$ are close, but the two quantities do not have to be equal [29. This agreement makes $\mathrm{Rb}_{3} \mathrm{C}_{60}$ look like one of the most simplistic materials. We have to keep in mind, however, that with $\lambda_{t r} \approx 1$ the mean free path of the electrons is actually shorter than the lattice spacing. The existence of metallic conduction with such a short mean free path remains one of the mysteries of the transport in alkali fullerides.

The second conclusion concerns the characteristic phonon frequency. It turns out to be $\omega_{0} \approx 210 \mathrm{~cm}^{-1}$, which is larger than the typical frequency of the acoustic phonons. This result is in agreement with the photoemission studies and calculations of Gunnarson et al. [4], where the low lying Raman modes of the $\mathrm{C}_{60}$ molecule proved to contribute significantly to the electron - phonon coupling.

In summary, we investigated the energy gap of the superconducting state of $\mathrm{Rb}_{3} \mathrm{C}_{60}$ and we demonstrated that this material cannot be described by the (weak coupling) BCS theory. The dimensionless electron - phonon coupling constant and the typical phonon frequency were determined. It is expected that the Eliashberg theory, with a coupling constant in the order of 1 , fully describes the superconductivity this material.

\section{ACKNOWLEDGMENTS}

We are indebted to P.B. Allen for enlightening discussions, to G.P. Williams and G.L. Carr for valuable advice during the measurements at the NSLS, and to S. Lindaas and C. Jacobsen for the use of the scanning atomic force microscope. This research has been supported by the Swiss National Science Foundation, by the NSF grants DMR-9501325 and INT-9414840, and by the Hungarian National Science Foundation grant OTKA-T015552. The NSLS and the U4IR beamline is supported by the US Department of energy, under the grant DEFG-0291ER4531.

* Permanent address: Institute of Physics, Technical University, Budapest, Hungary
** Permanent address: Research Institute for Solid State Physics, Budapest 1525, Pf. 49, Hungary

[1] P. B. Allen and R.C. Dynes, Phys. Rev. 12, 905 (1975)

[2] J.P. Carbotte, Rev. Mod. Phys. 62, 1027 (1990)

[3] W.L. McMillan, Phys. Rev. B 167, 331 (1968)

[4] O. Gunnarson et al., Phys. Rev. Letters 74, 1875 (1995); C.M. Varma, J. Zaanen, and K. Raghavachari, Science, 254, 989 (1991); M.Schluter et al., Phys. Rev. Letters, 68, 526 (1992)

[5] L.D. Rotter, Z. Schlesinger, J.P. McCauley, N. Coustel, J.E. Fischer, A.B. Smith, Nature, 355, 532 (1992)

[6] S.A. FitzGerlad, S.G. Kaplan, A. Rosenberg, A.J. Sievers, R.A.S. McMordie, Phys. Rev. B 45, 10165(1992)

[7] L. Degiorgiet al., Phys. Rev. B 49, 7012 (1994)

[8] L. Degiorgi, G. Briceno, M.S. Fuhrer, A. Zettl and P. Wachter, Nature 369, 541 (1994)

[9] Z. Zhang et al., Science, 254, 1619 (1991) C.-C. Zhang et al. Nature, 353333 (1991)

[10] P. Jess, U. Hubler, S. Behler, V. Thommen-Geiser, H.P. Lang, H.J. Güntherodt, Synth. Met., 77, 201 (1996)

[11] Chun Gu, et al., Phys. Rev. B 50, 16566 (1994)

[12] Tycko et al., Phys. Rev. Letters, 68, 1912 (1992)

[13] V.A. Stenger, C.H. Pennington, D.R. Buffinger, R.P. Ziebarth, Phys. Rev. Letters, 74, 1649 (1995)

[14] R.F. Kiefl et al., Phys. Rev. Letters, 70, 3987 (1993)

[15] W.A. Vareka, A. Zettl, Phys. Rev. Letters, 72, 4121 (1994)

[16] For a summary of the intramolecular modes, see M.C. Martin et al., Phys. Rev. B 50, 173, (1994).

[17] D. Koller, M.C. Martin, P.W. Stephens and L. Mihaly, Rev. Sci. Inst. 65, 760 (1994)

[18] M.C. Martin, D. Koller, X.Q. Du, P.W. Stephens, and L. Mihaly, Phys. Rev. B 49, 10818 (1994)

[19] T.T.M. Palstraa, R.C. Haddon, A.F. Hebard, J. Zaanen, Phys. Rev. Letters, 68, 1054 (1992)

[20] R.E. Glover, M. Tinkham, Phys. Rev. 108243 (1957)

[21] D.C. Mattis and J. Bardeen, Phys. Rev. 111, 412 (1958)

[22] D. Mandrus, L. Forro, D. Koller and L. Mihaly, Nature, 351, 460 (1991)

[23] This formula accounts for the tunneling of single electrons (see, for example, M. Tinkham, Introduction to superconductivity McGraw-Hill, New York, 1975). The Josephson tunneling contributes to the DC current at zero bias. However, this contribution is typically small for large resistance junctions (V. Ambegaokar and A. Baratoff, Phys. Rev. Letters, 11, 104 (1963)).

[24] It remains to be seen why did the optical reflectivity study of Degiorgi et al., Ref. 8], yield a smaller gap. Nevertheless, in that measurement a downturn was seen in the reflectivity curve at the energy close to the $2 \Delta$ value found by us.

[25] P.B. Allen and D. Rainer, Nature, 349, 396 (1991)

[26] R.C. Dynes, V. Narayanamurti and J.P. Garno, Phys. Rev. Letters, 41, 1509 (1978)

[27] E.L. Wolf, Principles of Tunneling Spectroscopy (Clarendon, Oxford, 1988)

[28] R. Akis, C. Jiang and J.P. Carbotte, Physica C 176, 485 (1991)

[29] P.B. Allen et al., Phys. Rev. B 34, 4331 (1986) 